\title{
La política entre la guerra, la resistencia y la gubernamentalidad según Michel Foucault ${ }^{1}$
}

\author{
Una lectura topológica de las definiciones del concepto de \\ política de los cursos de fines de la década del 70
}

\section{The politics between war, resistance, and the governmentality according to Michel Foucault}

A topological reading of the definitions of the concept of politics of the courses at the end of the 70 s

Luis Félix Blengino ${ }^{2}$

Universidad Nacional de La Matanza. Argentina.

Recepción: 9 de agosto del 2018

Evaluación: 10 de septiembre del 2018

Aceptación: 22 de octubre del 2018

1 Este trabajo se produjo como conclusión del trabajo con el equipo perteneciente al Proyecto de Investigación CyTMA2-C2DER027: "El concepto foucaulteano de "Política" en la segunda mitad de la década del '70: el lugar del "derecho" entre la guerra, el gobierno y la resistencia", de la Universidad Nacional de La Matanza (UNLaM), Argentina.

2 Doctor en Ciencias Sociales (UBA) Profesor universitario de Filosofía (UBA). Docente investigador Adjunto del Departamento de Derecho y Ciencia Política (UNLaM) Ciudad Autónoma de Buenos Aires.

Correo electrónico: luis.blengino@gmail.com

Blengino, L. (2019). La política entre la guerra, la resistencia y la gubernamentalidad según Michel Foucault. Cuestiones de Filosofía, 4 (23), 47-74.

doi: https://doi.org/10.19053/01235095.v4.n23.2018.8981 


\section{Resumen:}

En este artículo propongo una vía de reconstrucción del concepto foucaulteano de la política para la que tomaré como hilo conductor las tres principales definiciones vertidas en los cursos de finales de los 70 . La interpretación topológica propuesta permitirá comprender las articulaciones entre las tres definiciones como dimensiones de un mismo concepto. Nuestra hipótesis es que la tercera definición, i.e. el juego entre gubernamentalidades heterogéneas, sobredetermina a las otras dos dimensiones del concepto, i.e. a la relación antagónica entre sujetos y al enfrentamiento de una forma de subjetivación con una gubernamentalidad. Con esta finalidad primero expondremos la diferencia entre relaciones de poder y política, luego analizaremos la hipótesis Nietzsche, para finalmente exponer las hipótesis acerca de la política como resistencia y de la política como relación estratégica entre artes de gobernar. Para concluir se extraerán algunas consecuencias para la teoría de la subjetivación política fundadas en esta comprensión de la política como un triángulo cuyo vértice de la relación estratégica entre gubernamentalidades heterogéneas sobredetermina la constitución del sujeto político de la resistencia y el antagonismo.

Palabras clave: Foucault, política, guerra, resistencia, gubernamentalidad.

\section{Abstract:}

In this article, we propose a road to rebuild the Foucault concept of politics that I will take as the central thread of the three main definitions that were imparted in his courses at the end of the $70 \mathrm{~s}$. The topological interpretation here proposed will allow understanding the links between the three definitions that work as dimensions of the same concept. Our hypothesis is that the third definition, i.e., the game between heterogeneous governmentalities overdetermines the two remaining dimensions of the concept, that is, the antagonistic relationship between subjects and the confrontation as a manner of uprising with the governmentality. Having this goal, we will first expose the difference between the relationship of power and politics and then, we will analyze the hypothesis of Nietzsche. Finally, we will show the hypothesis about the politics as a resistance and the politics as a strategic relationship among the arts of governing. To conclude, some consequences will be drawn to the theory of political subjectivization grounded in this understanding of the politics as a triangle which vertex of the strategic relationship between heterogeneous governmentalities overdetermine the constitution of the political subject of the resistance and antagonism.

Keywords: Foucault, politics, war, resistance, governmentality. 
La politique entre la guerre, la résistance et la gouvernementalité, selon Michel Foucault. Une lecture topologique des définitions du concept de politique des cours de la fin des années $\mathbf{7 0}$

\section{Résumé:}

Dans cet article, je propose une voie pour reconstruire le concept foucaldien de la politique, pour laquelle je prendrai comme fil conducteur les trois définitions principales qui ont été répandues dans les cours de la fin des années 70. L'interprétation topologique proposée permettra de comprendre les articulations entre les trois définitions comme dimensions d'un même concept. Notre hypothèse est que la troisième définition, c'est-à-dire le jeu entre gouvernementalités hétérogènes, surdétermine les deux autres dimensions du concept, à savoir la relation antagoniste entre sujets et la confrontation entre une forme de subjectivation et une gouvernementalité. Dans ce but, nous exposerons d'abord la différence entre les relations de pouvoir et la politique, puis nous analyserons l'hypothèse de Nietzsche, pour finalement exposer les hypothèses sur la politique comme résistance et sur la politique comme relation stratégique entre arts de gouverner. En conclusion, quelques conséquences seront tirées pour la théorie de la subjectivation politique basées sur cette compréhension de la politique comme étant un triangle dont le sommet de la relation stratégique entre des gouvernementalités hétérogènes surdétermine la constitution du sujet politique de la résistance et lıantagonisme.

Mots-clés: Foucault, politique, guerre, résistance, gouvernementalité.

\section{A política entre a guerra, a resistência e a gubernamentalidad segundo Michel Foucault. Uma leitura topológica das definições do conceito de político dos cursos ao final da década do 70}

\section{Resumo:}

Este artigo propor uma via de reconstrução do conceito foucaulteano da política como fio condutor das três principais definições nos cursos de finais dos 70 . A interpretação topológica proposta permitirá compreender as articulações entre as três definições como dimensões de um conceito mesmo. Nossa hipótese é que a terceira definição, i.e., o jogo entre gubernamentalidades, heterogéneas, sobredetermina as outras duas dimensões do conceito, i.e., à relação antagónica entre sujeitos e o enfrentamento de uma forma de subjetivação com uma gubernamentalidad. Com esta finalidade primeiro 
exporemos a diferencia entre relações de poder e política, logo analisaremos a hipóteses Nietzsche, para finalmente, expor as hipóteses sobre a política como resistência e da política como relação estratégica entre artes de governar. Para concluir, se extraíram algumas consequências para a teoria da subjetivação política fundadas nesta compreensão da política como um triangulo da relação estratégica entre gubernamentalidades heterogéneas sobre determina a constituição do sujeito político da resistência e o antagonismo.

Palavras-chave: Foucault, política, guerra, resistência, governamentalidade. 


\section{Introducción}

En la "Tercera Jornada" de El tiempo que resta, Agamben inicia un debate con Rancière en torno a la naturaleza del litigio y el daño, a partir del abordaje de la cuestión del pueblo en la encrucijada de Foucault con Marx. Sin embargo, lo que está en juego es la concepción de la política. Así -al tomar como punto de partida la idea de una relación dialéctica sin mediaciones que convoca a la parte, al todo y a un resto que implica un excedente respecto de ambos y que "significa la imposibilidad para el todo y la parte de coincidir consigo mismo o entre ellas" (Agamben, 2006, p. 60)- se propone problematizar el tipo de frontera que supone pensar lo político desde la noción de pueblo, entendido como plebe o resto.

En este contexto, el pensador italiano sugiere que Rancière ha desarrollado su filosofía política a partir de una reelaboración inadecuada del concepto de plebe esbozado por Foucault (cf. 1992, p. 177), en una entrevista realizada por el mismo filósofo franco-argelino en 1977. Se inicia así una controversia que llega hasta el presente en torno al concepto y al sujeto de la política en el pensamiento de Michel Foucault, en la medida en que la filosofía política de ambos epígonos se posiciona a partir de lo no dicho o lo solamente sugerido o esbozado por el filósofo de Poitiers.

Desde mi perspectiva, sin embargo, ambas lecturas resultan unilaterales y, en esa medida, si bien pueden ser de utilidad para desarrollar sus respectivas filosofías políticas, no así para comprender la del propio Michel Foucault. Por lo tanto, luego de presentar muy sucintamente la controversia entre ambos epígonos (Agamben y Rancière), me propongo reconstruir lo que denominaré el triángulo de la política, a partir de las tres principales definiciones foucaultianas de la política, tal como se delinean en los cursos de la segunda mitad de los años 70 .

Giorgio Agamben interpreta que la plebe foucaultiana sería un "elemento inasignable y absolutamente irreductible a las relaciones de poder" (2006, pp. ), en un sentido análogo al proletariado en Marx, que en cuanto víctima de "la injusticia absoluta" -y no de una injustica particular- emerge como el sujeto de la política revolucionaria. Por su parte, Rancière sostiene que el daño político, es decir, la injusticia que instituye la democracia y la política como comunidad de litigio, sería por definición "tratable". Esto, finalmente, terminaría por difuminar la frontera entre la democracia entendida como litigio por el reparto de las partes de la comunidad y la posdemocracia, o sea, el

Blengino, L. (2019). La política entre la guerra, la resistencia y la gubernamentalidad según Michel Foucault. Cuestiones de Filosofía, 4 (23), 47-74. 
régimen consensual de armonización de intereses. En este sentido, el filósofo italiano sostiene que la comprensión del litigio democrático supone, por lo tanto, una concepción del pueblo como víctima de una injusticia absoluta e intratable, lo cual implica asumir la posibilidad del enfrentamiento radical, es decir, "de una stasis, una 'revuelta', o de una guerra civil" (Agamben, 2006, pp. 62-63)

La respuesta de Rancière toma dos caminos argumentativos. Por un lado, afirma que la construcción teórica de una víctima absoluta de un "Mal infinito", a la que asistiría un derecho absoluto, es el correlato teórico más adecuado a los fines de una concepción de la política, que se sustenta en la convicción de que tal derecho debiera ser heredado por un defensor de aquella víctima impotente. De este modo, tal planteo entraría en consonancia, aunque con mediaciones inocultables, con la recientemente reconocida obligación ética universal y absoluta dirigida a la reparación infinita de un daño absoluto. Una convicción teórica que sirve, como es evidente, a su puesta en acto por las potencias imperiales a través de la denominada "intervención humanitaria" (Rancière, 2010, pp. 95-97).

En este sentido, de acuerdo con Rancière, la referencia de Bush -luego de los atentados del 11 de septiembre de 2001- a la "justicia infinita" frente a crímenes infinitos para invocar el derecho de intervención humanitaria, a partir del cual las potencias occidentales expandieron desde entonces sus zonas de injerencia, determina el contexto inmediato en el que debe situarse la teoría de Agamben. Asimismo, debería tenerse en cuenta, a los fines de la práctica política, que este tipo de teoría borraría del horizonte de expectativas cualquier esperanza en la emancipación humana (Rancière, 2004, p. 308), pues al "infinitizar la justicia” termina por considerar el daño como intratable políticamente, ya que implica aceptarlo como una suerte de destino ontológico frente al cual sólo se podría resistir.

Por el otro lado, al tomar distancia de las definiciones de aquella entrevista de juventud, Rancière no ha dejado de insistir en el hecho de que su objetivo teórico es fundamentalmente diferente del foucaultiano. Desde su perspectiva, el pensamiento político de Foucault se reduciría a lo que él mismo ha analizado bajo el concepto de orden policial, al que, de hecho, buscaría especificar $-\mathrm{y}$ reelaborar- a partir del concepto de posdemocracia. En consecuencia, Rancière interpreta que el estudio de la biopolítica, del cuerpo

3 La cursiva es del autor. 
y de las poblaciones como objetos del poder y no como sujetos políticos, ha constituido el verdadero interés teórico de Foucault, quien únicamente se habría ocupado del poder y no de la cuestión política. Por el contrario, lo que él mismo definió como política, es decir, la "forma de subjetivación política", a partir de la verificación del principio igualitario, no habría sido un objeto de preocupación teórica para el filósofo del poder (Rancière, 2000, p. s/n).

Este contrapunto permite recordar dos advertencias metodológicas que conviene tener en cuenta para reconstruir el concepto foucaultiano de política, puesto que, simultáneamente, hacen posible comprender la distancia que separa aquellos enfoques de la conceptualización foucaultiana de la política y a ésta de la reducción a la que es sometida por sus epígonos más eminentes.

En primer lugar, entonces, cabe subrayar la advertencia relativa a la importancia de ceñirse a la especificidad histórica para realizar un análisis político inmanente, lo que, a su vez, condiciona el pensamiento de las luchas y emancipaciones. En este sentido, cuando en Nacimiento de la biopolitica (2007) Foucault aborda lo que llama "el prejuicio fóbico del Estado", señala cierto valor inflacionario de la crítica inducida por esta perspectiva, que lleva al análisis a perder de vista la singularidad histórica de cada caso. En el ejemplo brindado por Foucault se hace inocultable la distancia que separa su enfoque no sólo del de Hayek y los neoliberales, sino también de perspectivas como la agambeniana. Tal es el caso de la advertencia contra los procedimientos teóricos a través de los cuales el análisis de la seguridad social, y los mecanismos de administración que la sostienen, se desplaza y remite:

(...) a partir de algunos deslizamientos y gracias al juego con algunas palabras, al análisis de los campos de concentración. Y de la seguridad social a los campos de concentración se diluye la especificidad -necesaria, sin embargodel análisis. Por lo tanto, inflación, en el sentido de que hay crecimiento de la intercambiabilidad de los análisis y pérdida de su especificidad (Foucault, 2007, pp. 219-220).

Tomando en cuenta esta advertencia, entonces, se comprende una de las diferencias clave entre el enfoque filosófico político de Foucault y la teoría de Agamben sobre el campo como nomos de la política moderna: el lugar que ocupa el Estado y el poder soberano. En Foucault, el concepto de gubernamentalidad como generalidad singular (Senellart, 2006, pp. 449-450) se orienta a enfocar la contingencia y reversibilidad de las relaciones políticas, al hacer entrar al Estado en un campo de análisis estratégico bajo el diagnóstico 
de un progresivo alejamiento de una gubernamentalidad de Estado, a partir del despliegue de una tendencia creciente hacia la gubernamentalización del Estado (Foucault, 2006, p. 136). En Agamben, por el contario, la genealogía parece identificar, de modo análogo a los neoliberales, la esencia del Estado con el Estado total, convertido en paradigma y destino de la política occidental fundada en la cooriginariedad de la soberanía y el biopoder.

La advertencia foucaultiana se refiere, en última instancia, a no perder de vista la singularidad histórica de la serie de los juegos políticos que no deberían pensarse bajo la perspectiva de una creciente "estatización de la sociedad", al recurrir a las ideas de un avance irresistible del Estado sobre la vida y de una resistencia de la vida ante un poder estatal que incesantemente pretende tomarla a su cargo.

En segundo lugar, hay que tener presente que una interpretación adecuada del concepto foucaultiano de la política debería ajustarse a otra advertencia que hace el filósofo en 1983, respecto al peligro de pensar la política desde la perspectiva heideggeriana, por decirlo retomando la tesis de Marchart (2007, pp. 35-60), de la diferencia ontológica. En efecto, con esta perspectiva, tendiente a identificar lo específicamente político con la ruptura, se corre el riesgo de subestimar la importancia del análisis político no sólo del orden y el gobierno, sino del juego político en torno a estos, lo que obliga a matizar el tipo de interpretaciones y reapropiaciones como las reseñadas. Al respecto, en la clase del 2 de febrero de 1981, Foucault señala lo siguiente:

Los problemas del poder, son en sentido estricto problemas de la política, y nada me parece más peligroso que ese famoso deslizamiento de la política a lo político utilizado en neutro ("lo" político), que en muchos análisis contemporáneos sirve, a mi juicio, para enmascarar el problema y el conjunto de problemas específicos que son los de la política, la dynasteia, el ejercicio del juego político, y de éste como campo de experiencia con sus reglas y su normatividad (Foucault, 2009, p. 171).

Siguiendo esta precaución, no parece ocioso subrayar, aunque sea evidente, que la distinción conceptual foucaultiana entre poder y política que está a la base, no puede coincidir con la topología posestructuralista sostenida en la distinción entre lo ontológico y lo óntico (Marchart, 2007, pp. 35-60).

Por último, antes de pasar a la reconstrucción del concepto foucaulteano de política, es posible señalar en qué sentido las interpretaciones de Rancière y Agamben resultan al menos incompletas. Por un lado, no se puede sostener que el filósofo de Poitiers identifique la problematización de la política con el 
estudio de las relaciones de poder, en el hecho de no haber fundado sus análisis en la distinción entre la política y "lo político"; y menos aún considerar que ello es sinónimo de no haberse interesado por el problema de la política y del ejercicio del poder político, pues se trataría más bien de lo opuesto, esto es, que el análisis político requiere un concepto de la política que no caiga en la trampa posestructuralista. Pero, por otro lado, tampoco es prudente interpretar la filosofía política foucaultiana desde una perspectiva aún presa del "prejuicio fóbico del Estado", para leerla a partir del clivaje vida-poder, lo que habilita la identificación entre política y biopolítica. En ambos casos, estamos ante reconstrucciones insuficientes y sesgadas del pensamiento de Foucault de fines de los 70, ciertamente más complejo y disperso, sobre la cuestión de la política.

\section{Poder y política. Algunas precisiones}

En principio, retomaré la idea según la cual, para Foucault, los problemas del poder son también los problemas de la política, con el fin de exponer la especificidad y las conexiones entre el concepto de relaciones de poder y el de política. Para ello, lo primero que hay que recordar es que, a juicio de Foucault, el poder no es una sustancia, sino que se trata de relaciones de poder entre individuos o grupos de individuos. Estas relaciones de poder son relaciones de gobierno estratégicamente orientadas a la conducción de la conducta del otro, a través de la determinación táctica del campo de su acción posible, en vistas a una serie de objetivos específicos. Se puede gobernar, en este sentido, a una población, a un grupo dentro de ella o a los individuos. El concepto de gubernamentalidad, en efecto, es acuñado por Foucault para estudiar las relaciones de poder, lo que en Nacimiento de la biopolítica queda explicitado del siguiente modo:

El término mismo de poder no hace otra cosa que designar un [ámbito] de relaciones que resta analizar por completo, y lo que propuse llamar gubernamentalidad, es decir, la manera de conducir la conducta de los hombres, no es más que la propuesta de una grilla de análisis para esas relaciones de poder (2007, p. 218).

Pero es también en el final de ese mismo curso que Foucault elabora una definición de la política como relación estratégica contingente entre artes de gobernar heterogéneas. Así, al hacer un uso específico de la idea de gubernamentalidad -entendida como racionalidad estratégica de gobierno y como conjunto de relaciones de poder, de tecnologías y técnicas que permiten 
el ejercicio efectivo del poder en coyunturas determinadas-Foucault enumera tres tipos generales cuyas relaciones estratégicas (instrumentales, lúdicas y bélicas) constituyen la especificidad de la política según la tercera de las definiciones que abordaré.

Por otra parte, en un manuscrito insertado entre las clases del mismo curso, la política es definida como la resistencia a una gubernamentalidad específica, es decir, como un antagonismo entre una forma de subjetivación y un conjunto de tecnologías o una racionalidad de gobierno. Esta definición -la segunda por abordar, pues seguiré un criterio cronológico para ello- se apoya también sobre aquella diferencia con las relaciones de poder existentes entre sujetos libres.

En relación con esto último, es necesario destacar que si bien Agamben pone en el centro de su interpretación de Foucault una oposición fundamental para comprender la problematización de la política en su obra, como es la establecida entre el pueblo y la población (Agamben, 2002, p. 84), no obstante, en su análisis esta oposición es reconducida al problema de lo que Foucault denominó gubernamentalidad de partido. En efecto, Agamben ha subrayado sobre todo la manera en que Foucault pensó la política como continuación de la guerra por otros medios (la primera definición que abordaré) y la deriva por la cual la guerra de razas se reconfiguró biopolíticamente en racismo de Estado $^{4}$.

Sin embargo, de la transformación fundamental de la modernidad a partir de la cual "cada pueblo se dobla en población, [y] cada pueblo democrático es, al mismo tiempo, un pueblo demográfico" (Agamben, 2002, p. 84), se desprende no sólo una gubernamentalidad racista fundada en una idea integrista del pueblo como la que se va dibujando en Defender la sociedad a través del estudio del proceso de "estatización de lo biológico" y de biologización de la guerra de razas (Foucault, 2000a, pp. 187-189, 209-215, 217-218 y 230-233), sino también otra idea de pueblo, entendido como sujeto de la resistencia

\footnotetext{
4 En El Reino y la Gloria (Agamben, 2008, pp. 132-137), por ejemplo, al retomar la cuestión de la cesura biopolítica que separa al pueblo de la población, hace fuerte hincapié en las analogías que existen entre la deriva biopolítica del discurso histórico de la guerra de razas -tal como resulta de la genealogía foucaultiana del racismo de Estado- y la teoría política schmittiana acerca del gobierno qua conducción de un movimiento popular. Agamben, al retomar las líneas fundamentales de Defender la sociedad, explica de este modo el hecho de que el desdoblamiento del pueblo en población no es más que la condición de posibilidad de una forma de clivaje de tipo racista con la que se reintroduce "en el sistema de 'hacer vivir' el principio de la guerra" (2002, p. 84).
} 
política al gobierno económico, que es enfáticamente subrayada por Foucault en el curso Seguridad, territorio, población (2006, pp. 63-66 y 101-102). Si el primer camino implica, evidentemente, una relación entre sujetos -el pueblo y la población sobre la que aquel decide- el segundo sugiere un movimiento de desujeción y contraofensiva respecto a ciertos mecanismos por los cuales se es constituido y gobernado como parte de una población ${ }^{5}$.

Sin embargo, antes de volver sobre este punto, es necesario detenerse en la primera de las definiciones.

\section{La política como guerra y el problema del sujeto político}

En 1976, Foucault propone definir la política como "continuación de la guerra por otros medios", invirtiendo la clásica sentencia de Clausewitz para sostener lo siguiente:

Ley, poder y gobierno son la guerra, la guerra de unos contra otros [y] la rebelión, por lo tanto, no va a ser la ruptura de un sistema pacífico de leyes por una causa cualquiera [sino] el reverso de una guerra que el gobierno no cesa de librar (2000a, p. 106).

Esta hipótesis bélica como grilla de inteligibilidad de la política, no del poder como suele interpretarse, fue puesta en cuestión a partir de 1977 para ser reinstalada en la nueva constelación conceptual dominada por la grilla de la gubernamentalidad hacia 1979. La política, al igual que la guerra, implicaría la relación (antagónica) entre sujetos, pero a diferencia de ésta supondría la puesta en marcha de otros medios para combatir al enemigo. Las armas en la

\footnotetext{
5 Estos dos sentidos de pueblo a los que referimos se adecuan a las dos nociones fuertes de política que estructuran el discurso foucaulteano tal como lo interpreta Marcelo Raffin en su reciente artículo (cf. 2018, pp. 31-33). Aunque podemos acordar con la descripción general que propone Raffin al marcar el contrapunto a través del cual la distinción fundamental entre poder y libertad reverbera en el concepto de política; sin embargo, no nos parece justificado afirmar que la nuestra ( $c f$. Blengino, 2017, pp. 4-23) sea una perspectiva que toma a la política como elemento parcial y disruptivo, cuando, antes bien, allí hemos pretendido describir y analizar la estructura singular del discurso histórico político foucaulteano que se delinea en los cursos de la segunda mitad de la década del 70. A partir de la consideración de las tres definiciones de política que operan en él y como continuación de tal trabajo, aquí, procuraremos argumentar en favor de la tesis de que entre ellas puede establecerse una relación conceptual que permite dar cuenta de un diagnóstico político del presente que toma por objeto las tres formas de relación política que operan en la situación concreta a ser estudiada. A saber, la relación bélica o antagónica entre sujetos históricos colectivos; la relación de resistencia de un sujeto colectivo determinado frente y/o contra una gubernamentalidad singular; las relaciones estratégicas entre tecnologías de poder y entre artes de gobernar heterogéneas en una situación histórica específica.
}

Blengino, L. (2019). La política entre la guerra, la resistencia y la gubernamentalidad según Michel Foucault. Cuestiones de Filosofía, 4 (23), 47-74.

doi: https://doi.org/10.19053/01235095.v4.n23.2018.8981 
lucha política ya no serían blancas o de fuego, sino las leyes e instituciones del orden civil, aunque el objetivo sería el mismo: combatir y dominar o exterminar al enemigo.

Ahora bien, esta grilla de inteligibilidad como es sabido choca contra el problema de la libertad y la constitución del sujeto en el antagonismo, o sea, con la cuestión del carácter polemógemo de las identidades políticas. En efecto, la hipótesis Nietzsche -como también se conoce a esta definición binaria de la política- no sólo permite sacar a la luz la dominación subyacente a cualquier orden político, sino también el problema de la subjetivación, esto es, o los sujetos preexisten a la lucha o sus identidades se constituyen en dicho antagonismo. En este sentido, como demuestra Nosetto, esta hipótesis permite señalar un dominio estratégico de articulación de las luchas en el que "ninguna articulación global se agota en la programación exhaustiva de un sujeto" (2014, p. 59), es decir, que abre una forma de problematización que se dirige hacia el cuestionamiento de "la codificación clasista como garantía última de la articulación de las luchas" (2014, p. 51).

En efecto, Nosetto (2014, pp. 56-58) explicita de qué forma -al dejar de concebir a la política como la lucha por el poder (del Estado) y comenzar a analizarla como estrategia global de coordinación de las relaciones de poder, que no suponen ni un sujeto predestinado históricamente o prefijado sociológicamente de esa lucha, ni una determinación en última instancia económica- Foucault abre la indagación al modo en que se produce una relación de producción recíproca "entre la estrategia que fija, conduce, multiplica, acentúa las relaciones de fuerza y la clase que se identifica como dominante" (Foucault, 2001, p. 307).

En esta línea argumentativa cabe recordar que ya en La voluntad de saber Foucault sostenía que una serie de relaciones de fuerza múltiple recorren el cuerpo social y que "una línea de fuerza general que atraviesa los enfrentamientos locales y los vincula" da lugar a "las grandes dominaciones [que] son los efectos hegemónicos sostenidos continuamente por la intensidad de todos esos enfrentamientos" (2000b, pp. 114-115), que no obstante nunca son el resultado de la programación de un sujeto que preexiste a tales efectos o, como concluye Nosetto, que constituyen "efectos que exceden siempre la intención de los sujetos que las pusieron en marcha" (2014, p. 59). Sin embargo, lo esencial de este primer concepto de la política es subrayar que ésta consiste en la relación antagónica entre dos sujetos. Lo cual acerca al Foucault de la guerra de razas del curso de 1976 al planteo de Schmitt sobre lo político, tal como sugiere Giorgio Agamben (2008, pp. 132-137 y 2005, p. s/n). 
Sin embargo, como se evidencia en la cita que abre este apartado y a partir de la reflexión de Nosetto, esta definición de la política como relación entre sujetos enemigos es inescindible del problema de los medios propiamente políticos utilizados en el combate, así como de la cuestión de la relación de dichos sujetos con tales medios, lo que abre el problema de la constitución del sujeto político en el antagonismo. En efecto, son estas cuestiones las que permiten comprender el desplazamiento hacia la segunda definición, según la cual la política no puede ser reducida a una relación de antagonismo entre sujetos o agrupamientos humanos en función de la distinción amigo-enemigo.

Antes bien, el problema del carácter polemógeno de estas identidades conduce a la necesidad teórica de elucidar los mecanismos políticos de construcción del sujeto de la política, es decir, el problema de la subjetivación política, para lo cual Foucault se abocará a pensar el problema del enfrentamiento al orden institucional, ya no como un medio para combatir al enemigo (que sería el objetivo último de la política según la primera definición), sino como el fin mismo de la política, respecto del cual el combate del enemigo sería ahora un medio.

\section{La política como resistencia y la cuestión de la subjetivación política}

En el "manuscrito sobre la gubernamentalidad" de 1979, Foucault va a retomar el intento de definir la política ampliando y problematizando el concepto elaborado en 1976. En efecto, al apartarse de la grilla bélica para centrarse en el concepto de gubernamentalidad, Foucault delimita y explicita la segunda definición del concepto de política, la cual citaré in extenso:

El análisis de la gubernamentalidad como generalidad singular implica que "todo es político". Tradicionalmente se atribuyen dos sentidos a esta expresión:

- Lo político se define por toda la esfera de intervención del Estado. [...] Decir que todo es político es decir que el Estado está en todas partes, directa o indirectamente.

- Lo político se define por la omnipresencia de una lucha entre dos adversarios [...]. Esta otra definición es la de K. [sic] Schmitt. La teoría del camarada. [...]

En suma, dos formulaciones: todo es político por la naturaleza de las cosas; todo es político por la existencia de los adversarios.

Se trata, antes bien, de decir: nada es político, todo es politizable, todo puede convertirse en política. La política es, ni más ni menos, lo que nace 
con la resistencia a la gubernamentalidad, la primera sublevación, el primer enfrentamiento (Foucault, 2006a, p. 451).

La primera posición descartada hay que entenderla a la luz de aquel prejuicio compartido por Nietzsche, Marx, el neoliberalismo y la Escuela de Frankfurt (Foucault, 2006, pp. 136 y 2007, pp. 94 y 134) que Foucault identifica como "fobia al Estado", de la que hemos señalado que Agamben no se distancia. Este prejuicio fóbico del Estado postula una teleología expansiva y negativa inmanente al Estado que regiría su crecimiento indefinido, al aumentar los peligros que conlleva su misma esencia para la libertad y la seguridad de todos y cada uno.

Este prejuicio que toma al Estado, en palabras de Nietzsche, como un "monstruo frío", está en el corazón del error de un diagnóstico del presente que ha atravesado a una pluralidad de sistemas de pensamiento y que ha tenido un alto costo para las izquierdas: la sospecha o la certeza de que nuestra actualidad estaría marcada por el peligro de una "estatización de la sociedad", lo que lleva a pensar la política como el ejercicio del poder estatal sobre la vida o la sociedad identificándola con la biopolítica, cuya contraparte es concebir la resistencia como el enfrentamiento contra el Estado por parte de las fuerzas vivas de una sociedad que no se deja nunca apresar completamente por el orden burocrático-estatal.

La segunda perspectiva rechazada constituye la única referencia que Foucault hace al jurista alemán en su obra publicada hasta el momento. Si bien la síntesis propuesta no está exenta de problemas, es claro que la distancia marcada por Foucault es respecto de la lógica antagónica amigo-enemigo y sus consecuencias, es decir, la omnipresencia de una lucha que presupone y requiere de la existencia de los adversarios, aun cuando sea cierto que Carl Schmitt no identifica su propia teoría como una forma de adhesión a la sentencia "todo es político", pues sostiene que "la equiparación de 'estatal' y 'político' es incorrecta y errónea”, en tanto que la misma sólo pertenece al Estado total para el cual todo es político (Schmitt, 2004, p. 174).

En este sentido, también hay que recordar que para el jurista lo político qua intensidad extrema de la relación amigo-enemigo- podría muy bien inscribirse en el horizonte de la idea de que nada es por su propia naturaleza político, sino que todo es politizable. En efecto, que lo político pueda extraer su fuerza de los más diversos sectores de la vida humana, abre la posibilidad de pensar las condiciones de la construcción política de la identidad popular. 
Sin embargo, la distancia que marca Foucault respecto de esta perspectiva no apunta, como ya sugerí, sólo a señalar en la dirección de las condiciones de la constitución del sujeto político en el antagonismo, sino sobre todo a subrayar que este proceso de subjetivación política debe pensarse a partir de la relación de enfrentamiento con una gubernamentalidad determinada -ya sea en su totalidad o contra ciertos aspectos suyos-y no primariamente con un enemigo en una lucha omnipresente.

Por lo tanto, sólo en la medida en que la plebe es el "blanco constante y constantemente mudo de los dispositivos de poder", es decir, en que es una forma de ser relativa a un dispositivo y no a otro sujeto, es que lo plebeyo -lo que hay de la plebe en los cuerpos, los individuos y los grupos, para retomar la idea de 1977 que originó el debate entre Agamben y Rancière (Foucault, 1992, p. 177)- puede adoptar la forma del contragolpe, esto es, del enfrentamiento con tales dispositivos. En consecuencia, la resistencia política consiste ante todo en una relación del sujeto con los mecanismos de poder, en una forma de subjetivación contra cierto orden de cosas y no fundamentalmente -como se desprende del concepto de rebelión mentado en la primera definición- contra quienes son identificados como enemigos por defender o sostener activa o pasivamente dicho orden.

La forma de subjetivación política que pretende delinear Foucault con esta segunda definición es la que se produce en el enfrentamiento con las coordinaciones estratégicas de las relaciones de poder que conforman las grandes hegemonías sociales (siempre contingentes e inestables) que ella tiene por finalidad combatir y cambiar, para lo cual el combate de un sujeto identificado como enemigo, sea sustancial o circunstancial, es sólo un medio. Es decir, que la relación amigo-enemigo es, desde esta perspectiva, secundaria respecto de la relación sujeto-dispositivo. Al respecto, no está de más recordar la caracterización foucaultiana de los "movimientos de contraconducta" durante el curso de 1978:

Son movimientos cuyo objetivo es otra conducta, es decir: querer ser conducidos de otra manera, por otros conductores y otros pastores, hacia otras metas y otras formas de salvación, a través de otros procedimientos y otros métodos. Y son además movimientos que procuran -eventualmente, en todo caso- escapar a la conducta de los otros y definir para cada uno la manera de conducirse (Foucault, 2006a, p. 225).

A la luz de esto, se comprende que la política -entendida como lo que nace con el enfrentamiento a la gubernamentalidad a partir de la constitución 
de un movimiento de contraconducta, o sea, como forma de resistencia a una gubernamentalidad específica, de enfrentamiento con una hegemonía social determinada y como práctica de desujeción, desidentificación y subjetivación- supone a la vez que todo es político en cuanto forma parte de una gran hegemonía social, aunque también que nada lo es hasta el momento en que es cuestionado o combatido por una forma de subjetivación política.

Vale la pena aquí retomar lo apuntado sobre la cuestión de la plebe en "Poderes y estrategias", antes de referirme a la oposición entre pueblo y población. Foucault sostiene que si bien, evidentemente, no existe la realidad sociológica de la plebe, existe no obstante "algo" que escapa a las relaciones de poder, que es "el movimiento centrífugo, la energía inversa, lo no apresable" por ellas $^{6}$. Esa plebe no es tanto lo exterior al poder, sino "su límite, su anverso, su contragolpe" y en cuanto tal, subrayémoslo con énfasis, lo que "motiva todo nuevo desarrollo de las redes de poder" (1992, p. 177) 7 .

Sin embargo, para los fines de este artículo, sólo me interesa retener las dos ideas que permiten iluminar la definición que nos ocupa en este apartado: a) la plebe, qua lo que resiste y se opone al poder no puede ser identificado como algo dado que preexiste a la relación antagónica, sino que se constituye

6 En este sentido, recordemos que el filósofo afirma que "'La plebe no existe sin duda, pero hay de la plebe en los cuerpos, en los individuos y en los grupos" (Foucault, 1992, p. 177).

7 Como se deriva claramente de esta referencia, y si lo quisiéramos traducir en el lenguaje de la controversia entre Rancière y Agamben, lo plebeyo, tal como lo entiende Foucault, implicaría un daño tratable. No obstante, aceptar esto no implica en el marco del pensamiento foucaultiano, como mostraré, la imposibilidad de comprender -como argumentaba Agamben contra Rancière- el problema de la política, sino su condición de posibilidad. En efecto, asumir esta perspectiva analítica -según la cual cabe suponer que "hay 'de la' plebe" como anverso y contragolpe del poder- no puede, como apunta Foucault, "confundirse de ninguna manera con un neopopulismo que subtantificaría la plebe o con un neoliberalismo que cantaría sus derechos primitivos". Esta precisión es importante no sólo porque permite comprender que la plebe, tal como señala Brossat (1994, p. 143-145), se presenta ante todo como una función o un rol que cumple un personaje variable antes que un sujeto predestinado de la historia, sino también porque, según pienso, tanto la cuestión de un neopopulismo no substancialista, es decir, como forma de articulación política y constitución de una identidad colectiva, como la de un neoliberalismo no naturalista ni humanista, esto es, como una forma de liberalismo positivo, estarán, exactamente, en el centro de las reflexiones políticas foucaultianas de los textos sobre Irán (2001b, pp. 716 y 717, y 746 y 747) y del curso de 1979, respectivamente. Es decir, que esta idea de plebe es una de las claves de una grilla de inteligibilidad política que permite analizar uno de los clivajes capitales de este inicio de siglo XXI, como es el que existe entre una gubernamentalidad neoliberal y una populista, algo que parecen no tener en cuenta sus dos eminentes epígonos, en la medida en que no vinculan esta segunda definición con la tercera, asociada con el antagonismo entre racionalidades gubernamentales. 
en ella; b) el enfrentamiento no refiere principalmente a un enemigo, sino a determinados dispositivos y relaciones de poder que deben ser desmontadas, combatidas o neutralizadas. En suma, para Foucault no puede aceptarse la perspectiva de un sujeto predestinado de la historia ni previamente dado del conocimiento y la acción, así como tampoco la toma y/o la destrucción del Estado - qua lugar del poder- como el fin de la acción política -aunque sí como un objetivo táctico fundamental- como tampoco debería pensarse la relación política a partir de la prioridad de la lógica antagónica amigoenemigo, sino bajo una lógica estratégica y antagónica poder-resistencia, es decir, como una relación entre un dispositivo y lo que hay 'de la' plebe.

Para abonar esta interpretación, vale la pena recordar la oposición foucaultiana entre el pueblo y la población. Como es sabido, Foucault lleva a cabo la genealogía de la tecnología de poder fisiocrática, de sus objetivos, estrategias y programas, a través de un texto de cuya importancia advierte cuando lo presenta (2006, pp. 55-56) y que vuelve a enfatizar hacia el final del curso, al punto de afirmar que todo el análisis ha girado en torno a él (2006, p. 391). El texto es de Louis-Paul Abeille, data de 1763 y se titula Lettre d'un négociant sur la nature du commerce des grains. A partir de su estudio, Foucault señala que mientras que bajo el modelo disciplinario mercantilista la escasez y la carestía eran fenómenos indeseables que era preciso evitar, para la fisiocracia se trataría de fenómenos naturales que habría que gestionar en su naturalidad misma a través de la grilla de inteligibilidad económica de la oferta y la demanda. Esto marcaría, según Foucault, el nacimiento del dispositivo de seguridad por oposición al mecanismo jurídico-disciplinario del Estado de Policía o gubernamentalidad en la razón de Estado.

En efecto, desde la perspectiva fisiocrática, la escasez será percibida como una "quimera", siempre que se dejen actuar las fuerzas naturales de la oferta y la demanda en un marco de libre circulación de los granos y tomando a la población como el nivel pertinente del análisis (2006, pp. 62-63). Así, "la escasez como flagelo desaparece, pero la penuria que hace morir a los individuos no sólo no desaparece sino que no debe desaparecer" (Foucault, 2006, p. 63). Es en este contexto de análisis - del modo en que el surgimiento de los dispositivos de seguridad vinculados al gobierno económico fisiocrático convierten al pueblo en población gobernable a través de dichos dispositivosque Foucault subraya la centralidad de la oposición entre el pueblo y la población, no sólo porque el primero aparece como el sujeto de la resistencia a la biopolítica y los dispositivos de seguridad que le son correlativos desde el momento inicial del proceso de tendencial hegemonía biopolítica, sino 
también porque el pueblo emerge como el sujeto de una relación antagónica del tipo que nos interesa en este apartado. En este sentido, cabe recordar el pasaje central de esta problematización:

En ese dibujo que comienza a esbozar la noción de población también vemos perfilarse una partición en la cual el pueblo aparece de manera general como el elemento resistente a la regulación de la población, el elemento que trata de sustraerse al dispositivo por cuyo conducto la población existe, se mantiene y subsiste, y lo hace en un nivel óptimo. La oposición pueblo/población es muy importante. [...] no es similar a la oposición sujeto obediente-delincuente (2006, pp. 65-66).

El pueblo -i.e. el sujeto político que aparece como la contrafigura del sujetoobjeto político que es la población $(2006$, p. 63) - se caracteriza en el texto analizado por Foucault no como el sujeto de un antagonismo contra el enemigo del pueblo (tal como sería para Schmitt), tampoco como el sujeto soberano que decide sobre la población (tal como lo piensa Agamben), sino como el sujeto de una "inquietud" en una coyuntura creada por una forma de ser gobernado. En este contexto, "pueblo" es la práctica de subjetivación de quienes buscan sustraerse al dispositivo de seguridad a través del cual se pretende gobernarlos. El pueblo, entonces, constituye una forma de acción ante un dispositivo de gobierno económico y biológico, una forma de desujeción que -en la medida en que "está compuesto por aquellos que, en cuanto pueblo que se niega a ser población, van a provocar el desarreglo del sistema" (2006, p. 64)- implica simultáneamente una forma de subjetivación resistente en un contexto específico ante una gubernamentalidad determinada.

Se comprende, entonces, el alcance del desplazamiento que lleva de la primera definición de la política -como continuación de la guerra por otros medios y que suponía la idea de una relación entre sujetos antagónicos-a la segunda definición, de acuerdo con la cual la política es el movimiento de resistencia contra una gubernamentalidad, es decir, una forma de relación antagónica entre un sujeto y la serie de dispositivos por los cuales se lo pretende gobernar. En consecuencia, desde la perspectiva abierta por esta segunda definición, es evidente que el objetivo estratégico de la política no es combatir y/o eliminar al enemigo -lo cual se revela, a lo sumo, como un objetivo táctico- sino enfrentar, neutralizar, sustraer, desarreglar y/o cambiar una gubernamentalidad específica.

Ahora bien, dicha subjetivación política, ese devenir pueblo, eso que hay de la plebe, ese movimiento de sustracción defensiva y ofensiva frente a los 
dispositivos y tecnologías de gobierno, funciona como un movimiento de contraconducta, es decir, que a la vez que implica un procedimiento por el cual, a decir de Foucault, "la subjetividad (no la de los grandes hombres, sino la de cualquiera) se introduce en la historia y le da un soplo" (Foucault, 1999, p. 206), tiene por objetivo otra conducta, otra forma de conducción. Esta forma de subjetivación, como señalé, no implica ni la omnipresencia de los enemigos -es decir, que no supone el recurso a un sujeto de la historia, ni a la idea de un enemigo sustancial- ni la identificación del Estado como el centro del cual deriva la opresión general sobre la sociedad, el individuo, el hombre o la vida. Antes bien, implica el recurso a una otra gubernamentalidad alternativa, como punto de apoyo para un contragolpe y un ocasional reemplazo de los dispositivos vigentes.

En efecto, aunque Foucault suele afirmar, de un modo que recuerda el pensamiento lockeano de la resistencia (Locke, 2002, pp. 160-161), que la sublevación es un "hecho" y que "hay" de la plebe, ésta es el emergente de un juego de fuerzas histórico en el que una forma de subjetivación adquiere su singularidad fijándose los objetivos tácticos y estratégicos en una lucha específica contra una forma concreta de ser gobernado. En esa circunstancia cobra relevancia el acervo histórico de las artes de gobierno, de las memorias de las batallas y los saberes descalificados a los que se dirige la genealogía como método y arma de lucha, tal como es definida al inicio de Defender la sociedad (Foucault, 2000a, pp.20-23).

Es a partir de esto que adquiere sentido la tercera definición de la política, la que surge del trabajo del genealogista, en la medida en que éste muestra la emergencia de gubernamentalidades heterogéneas, su constitución en el combate, el debate y el juego de las tecnologías, los dispositivos y las técnicas que a la vez funcionan como punto de apoyo para las subjetivaciones resistentes, en cuanto el campo de sus prácticas está circunscripto por un juego de fuerzas históricas en el que es crucial la serie histórica de las racionalizaciones del ejercicio del gobierno de los hombres.

\section{La política como relación estratégica entre gubernamentalidades heterogéneas y la cuestión del triángulo de las artes de gobernar}

El curso de 1979 se cierra con la siguiente pregunta retórica dirigida al auditorio del Collège de France: “QQué es la política, en definitiva, si no el juego de esas diferentes artes de gobernar con sus diferentes ajustes y, a la vez, el debate que ellas suscitan? Es ahí me parece, donde nace la política" 
(2007, p. 358). Desde mi perspectiva, esta pregunta postula una definición que aparece a modo de conclusión de un argumento que puede ser reconstruido, al tomarla como la hipótesis que el curso se ha ocupado en demostrar. Por lo tanto, antes de detenernos en esta tercera definición, es necesario reconstruir el argumento foucaultiano considerándolo como la conclusión final de una indagación histórica cuyo diagnóstico general es que nuestra actualidad es una época que ha ingresado en una profunda crisis de gubernamentalidad, crisis comparable con aquella que dio origen a nuestro presente histórico durante el renacimiento, cuando en el siglo XVI "explotó" el problema de las conductas (2006, pp. 263 y 268).

Por otra parte, dicho diagnóstico apunta a señalar que nuestra contemporaneidad se caracteriza por lo que Foucault denomina como el "proceso de gubernamentalización del Estado" (2006, pp. 135 y 137). Como señalé, tal proceso es analizado en los cursos del 78 y 79 a través del recurso a una lógica estratégica de conexión de elementos heterogéneos que permanecen heterogéneos ${ }^{8}$. Queda así delineado un triángulo tecnológico (soberanía-disciplina-gobierno) que permite explicar la tendencia de gubernamentalización del Estado, es decir, la constitución del otro triángulo (seguridad-economía política-población) a partir de la dominancia de las modernas tecnologías de gobierno desde el siglo XVIII, cuando la ratio gubernamental liberal desplazó al dispositivo policial disciplinario propio de la razón de Estado (2006, pp. 136-137).

Desde entonces, según la genealogía foucaultiana, la dominante siguió la pendiente de alejamiento de la gubernamentalidad de Estado 9 . No obstante,

\footnotetext{
Esta perspectiva analítica toma como punto de partida la distinción y oposición entre la lógica dialéctica y la lógica de la estrategia. Mientras la lógica dialéctica procura hacer valer términos contradictorios en el elemento de lo homogéneo al prometer su resolución en una unidad, la lógica estratégica establece conexiones posibles entre términos heterogéneos que permanecen heterogéneos. En efecto, según el pensador de Poitiers la heterogeneidad "nunca es un principio de exclusión o, si lo prefieren, la heterogeneidad jamás impide la coexistencia, ni la unión, ni la conexión" (Foucault, 2007, p. 62). Es a partir de esta idea que se puede llevar a cabo un análisis que haga valer la lógica de la estrategia, es decir, "la lógica de la conexión de lo heterogéneo y no la lógica de la homogeneización de lo contradictorio" (2007, p. 62). Sobre la clasificación del concepto de estrategia en la obra de Foucault, remitimos al análisis de Nosetto (2014, pp. 59-67) donde distingue los tres modos de entender la conexión de los elementos heterogéneos -instrumental, lúdico y bélico- de los dos tipos de orientaciones -las de poder y las de lucha- y de los dos niveles -local y global- en función de los cuales opera el concepto de estrategia.

9 Esto vale tanto para el liberalismo clásico y el neoliberalismo como para el Estado benefactor y los gobiernos de partido, a pesar de lo que postulan neoliberales como Agamben.
} 
como en aquélla no se trata del mero reemplazo de los elementos en una secuencia lineal y progresiva, sino de su conexión estratégica, nunca debe suponerse ni su simple desaparición, ni un arreglo cuasi-funcional entre ellos. En efecto, tal como señala Collier (2009), la introducción de la lógica estratégica permite escapar a la solución cuasi-funcional y "totalizante", para pensar en términos de contingencia la relación entre las tecnologías y la constitución de las diferentes racionalidades gubernamentales y realizar así un "análisis topológico del poder" (Collier, 2009, p. 79). Esto explica que, en los cursos del 78 y 79, Foucault se proponga llevar a cabo:

(...) la historia mucho más global, pero desde luego también mucho más vaga, de las correlaciones y los sistemas de dominantes que hacen que, en una sociedad dada y para tal o cual sector específico - pues las cosas no siempre van a evolucionar forzosamente al mismo ritmo en uno $u$ otro sector, en un momento, una sociedad o un país determinados-, se introduzca, por ejemplo, una tecnología de seguridad que hace suyos y pone en funcionamiento dentro de su propia táctica elementos jurídicos, elementos disciplinarios, y a veces llega a multiplicarlos (Foucault, 2006, p. 24).

Es este análisis global de las conexiones estratégicas, i.e. contingentes, entre las tecnologías el que permite pensar la politicidad inherente al campo histórico, o mejor aún, permite pensar la política como una relación inmanente a la historia de las correlaciones y los sistemas de dominantes. De aquí que la idea de gubernamentalidad no pueda comprenderse como una estructura, i.e. una invariante relacional entre variables, sino como una "generalidad singular", es decir, una racionalidad estratégica cuyas variables establecen relaciones aleatorias que responden a coyunturas contingentes y no a secuencias históricas determinadas, lo que legitima un análisis estratégico, i.e. político (Senellart, 2006, pp. 449-450).

Es bajo esta clave de lectura que la historia de la gubernamentalidad ${ }^{10}$, desarrollada por Foucault en los cursos de 1978 y 1979, se abre a una comprensión posmarxista que la ilumina como el despliegue de conexiones (de correlación y dominancia) contingentes entre los dispositivos que conforman el triángulo soberanía-disciplina-seguridad (gobierno) en la época presente, marcada por la tendencia a la gubernamentalización del Estado. En efecto, este análisis global de las conexiones estratégicas entre tecnologías

10 Para saber más sobre el uso del concepto "historia de la gubernamentalidad", en el marco de la obra de Foucault, remitimos a Castro-Gómez (2012, pp. 9-15).

Blengino, L. (2019). La política entre la guerra, la resistencia y la gubernamentalidad según Michel Foucault. Cuestiones de Filosofía, 4 (23), 47-74. 
heterogéneas es el que permite comprender la política en cuanto relación inmanente al campo histórico.

$\mathrm{Y}$ es en este sentido que las relaciones estratégicas entre tecnologías constituyen el objeto de esa forma de indagación histórica y política a partir de la cual hacia el final del curso Nacimiento de la biopolítica, el triángulo de las tecnologías es reinterpretado políticamente por Foucault a la luz de la historia de la gubernamentalidad como un triángulo conformado por artes de gobernar heterogéneas: un arte de gobernar en la verdad, un arte de gobernar en la racionalidad del Estado soberano, un arte de gobernar en la racionalidad de los gobernados (2007, pp. 357-358).

Desde esta perspectiva, el traspaso del umbral de modernidad biológica, es decir, la ruptura con el orden precedente y el ingreso a la modernidad -i.e. a "las formas modernas de la tecnología gubernamental" (2007, p. 357)- se explica como el tránsito desde un tipo de gobierno ajustado a la sabiduría y la verdad hacia uno ejercido en conformidad con un principio de cálculo racional que dio lugar a formas gubernamentales sucesivas, ajustadas a la racionalidad instrumental, ya sea la del Estado soberano, ya sea la de los gobernados qua sujetos de interés.

Sin embargo, al igual que lo que ocurre -desde la perspectiva de las técnicas y tecnologías- con los elementos jurídicos y disciplinarios, desde la óptica de la historia de la gubernamentalidad, ni el arte de gobernar en la verdad -hegemónico en la era de la soberanía- ni el arte de gobernar en racionalidad del Estado soberano - propio de la primera modernidad-habrían sido reemplazados sin más, sino que constituirían gubernamentalidades heterogéneas a la dominante, que guardan diversas relaciones con ella, tal como lo señaló recientemente Ewald (2016 $)^{11}$. Es a partir de esta configuración triangular que Foucault va a pensar la politicidad como lo propio de la relación estratégica entre las diversas artes de gobernar.

En consecuencia, las grandes hegemonías sociales constituidas por las correlaciones estratégicas-de dominantey subordinadas-entre las tecnologías

11 Esto explica por qué el marxismo, por ejemplo, aparece en esta genealogía como constituyendo un intento de ajustar el gobierno a la racionalidad de una historia que se manifiesta progresivamente como verdad, o el Estado de bienestar que -recordémoslo, a contrapelo de lo que sostienen tanto los neoliberales como Agamben, en el relato histórico foucaultiano no es más que una variante de la gubernamentalidad liberal en la racionalidad de los gobernados-al recurrir a políticas nacionalistas se explica a partir del acople de su racionalidad propiamente liberal con un tipo de racionalidad ajustada a los intereses del soberano (2006, p. 358). 
de poder, es decir, la gubernamentalidad qua generalidad singular, aparece sobredeterminada por la serie de relaciones estratégicas -instrumentales, lúdicas y bélicas- entre artes de gobernar heterogéneas. En efecto, se comprende así que el diagnóstico de 1978, según el cual nuestro presente se halla marcado por la tendencia a la gubernamentalización del Estado iniciada hacia el siglo XVII, es decir, por la línea de fuerza que se despliega a partir de la emergencia de una tecnología de gobierno estructurada por la serie seguridad-economía-población y cuyo objetivo es la administración eficiente de la sociedad (Foucault, 2006, pp. 136-137), sea complementado con el diagnóstico de 1979, que apunta a destacar que a la historia de las tecnologías, entendida como las diferentes relaciones estratégicas que se establecen al interior del triángulo soberanía-disciplina-gobierno, le corresponde, desde el punto de vista de la historia de la gubernamentalidad, la serie de las relaciones estratégicas entre las tres artes de gobernar heterogéneas.

Este es el contexto general que enmarca los últimos párrafos de Nacimiento de la biopolítica, en los que Foucault vuelve sobre la idea de una historia contingente que, lejos de estar regida por una lógica lineal y cuasifuncionalista del abandono y el simple reemplazo, se despliega a partir de las relaciones de dominancia y subordinación entre tecnologías heterogéneas sobredeterminadas por el juego, el debate y el combate entre racionalidades programáticas de gobierno alternativas ${ }^{12}$.

Esto explica que, como conclusión de la serie de los cursos de fines de la década del 70 sobre la historia de la gubernamentalidad, Foucault introduzca bajo la forma de pregunta aquella tercera definición de la política como lo que nace del juego de ajustes, de combates y rebates y del debate que suscitan las superposiciones coyunturales de las "distintas maneras de calcular, racionalizar, regular el arte de gobernar" (2007, p. 358). De acuerdo con esto, la historia de la gubernamentalidad queda definida como intrínsecamente

12 En este sentido, luego de señalar que "la transformación importante" que había tratado de localizar a lo largo del curso era la operada por la racionalidad liberal de gobierno en la singularidad de su emergencia y despliegue estratégico - esto es, en cuanto la novedad de su problematización del arte de gobernar reside en la pregunta por cómo regular el gobierno implantando un principio de racionalización del arte de gobernar que se funda en el comportamiento racional de los gobernadosFoucault se ocupa de destacar que, no obstante esta emergencia y la ruptura que implica, focalizar en el punto de división "no significa -lejos de ello- que la racionalidad del Estado-individuo o del individuo soberano que puede decir 'yo, el Estado' sea abandonada"; y asimismo, "podrá decirse que el gobierno que se ajusta a la verdad no es tampoco algo que haya desaparecido" (2007, pp. 357-358).

Blengino, L. (2019). La política entre la guerra, la resistencia y la gubernamentalidad según Michel Foucault. Cuestiones de Filosofía, 4 (23), 47-74.

doi: https://doi.org/10.19053/01235095.v4.n23.2018.8981 
política, en cuanto su objetivo consiste en cartografiar esos juegos, debates y combates entre gubernamentalidades heterogéneas y alternativas y al interior de ellas.

En este sentido, la política entendida como relación estratégica aparece a partir de estas especificaciones del siguiente modo: a) como una relación instrumental de ajustes, apoyos y encabalgamientos tecnológicos que delinean estrategias de conjunto regidas por una relación de dominantesubordinadas vinculadas a la idea de gubernamentalidad qua "generalidad singular" y al triángulo de las tecnologías de poder; b) como una relación lúdica de competencia de acuerdo con las ideas de juego y debate, ya sea entre artes o racionalidades alternativas, ya sea en torno de la evaluación de tecnologías heterogéneas en términos de la relación medios-fines; c) como una relación bélica de confrontación, de acuerdo con las ideas de combate, enfrentamiento y rebate entre artes o racionalidades alternativas.

Para comprender estas especificaciones, conviene retomar el análisis que Luciano Nosetto (2013, p. 59) lleva a cabo del concepto de estrategia, tal como aparece en "El sujeto y el poder" (2001a) de 1982, donde Foucault le dedica el último apartado a la relación entre relaciones de poder y relaciones de estrategia. Allí se comienza por distinguir tres usos de la palabra estrategia: a) el primero tiene un sentido general y alude a la racionalidad instrumental puesta a funcionar para alcanzar un objetivo determinado; b) el segundo tiene un sentido lúdico, según el cual se trataría del juego de "las representaciones calculadas recíprocamente enfrentadas" con el fin de obtener una ventaja; c) el tercero tiene un sentido bélico referido a los procedimientos puestos a funcionar para la neutralización y la desposesión de los instrumentos y medios de combate del oponente, con el objetivo de ponerlo en la situación de abandonar la lucha y así obtener la victoria.

De acuerdo con esto, la primera acepción -instrumental-permite comprender políticamente las racionalidades gubernamentales en términos de estrategias de coordinación que ponen en correlación tecnologías y técnicas. Se trataría, en este sentido, de la relación estratégico-política general entre tecnologías $\mathrm{y}$ artes de gobernar que configuran una gubernamentalidad qua generalidad singular. La segunda refiere a una relación de competencia entre racionalidades alternativas y tecnologías heterogéneas que enmarcan la conducta de los actores que a través de ellas persiguen el objetivo de obtener una ventaja, es decir, una posición dominante o hegemónica frente a sus adversarios, en un juego probablemente indefinido. Se trata de la relación de gobierno entre los 
sujetos, entendida como conducción de conductas, sobredeterminada por la relación lúdica entre las tecnologías y gubernamentalidades que permiten determinar el campo de la acción posible de los otros.

La tercera se refiere a una relación antagónica entre sujetos que necesariamente está mediada por la relación con las racionalidades de gobierno, las tecnologías y las estrategias del adversario. En efecto, la neutralización y privación de los instrumentos del oponente e imposición de los propios son, como señalamos, los objetivos de la confrontación. Se trata de la lucha por el control de los instrumentos de gobierno del otro. Por lo que, en primera instancia, no se trataría de una relación entre sujetos colectivos enemigos como en Defender la sociedad, sino de un sujeto colectivo particular que rebate y combate determinadas racionalidades de gobierno y tecnologías de poder, por medio de las cuales pasa el antagonismo con el sujeto enemigo. La relación entre los enemigos, entonces, está sobredeterminada por la disputa en torno de la neutralización y la imposición de tecnologías y artes de gobernar alternativas.

Por lo tanto, se comprende que la definición de 1979, según la cual la política es "el juego de esas diferentes artes de gobernar con sus diferentes ajustes y, a la vez, el debate que ellas suscitan" (2007, p. 358), permite delimitar un campo específico para el análisis político, que complejiza la perspectiva posestructuralista, que como señalaba Foucault en el Gobierno de sí y de los otros, al atender casi exclusivamente al momento de "lo" político y poner excesivo énfasis en el acontecimiento, la irrupción y la interrupción, "peligrosamente" enmascara los problemas de la política como campo de experiencia del ejercicio del juego político y del ejercicio del poder (2009, pp. 171-172). En este sentido, el ámbito de estudio demarcado por este tercer concepto de la política abre el análisis político a la comprensión de tres tipos relaciones políticas:

a) La relación, en sentido amplio e instrumental, de ajuste estratégico entre tecnologías de gobierno al interior de una racionalidad gubernamental; b) la relación lúdica, de competencia entre racionalidades de gobierno alternativas, entre tecnologías heterogéneas y entre los grupos que buscan recíprocamente condicionar sus conductas a través de ellas, así como el debate que se desarrolla en torno de estas relaciones de ajuste y competencia; c) la relación de oposición y enfrentamiento entre artes de gobernar, las que dan lugar a las relaciones de resistencia a la gubernamentalidad, al enfrentamiento, la sublevación, es decir, a la relación de oposición y confrontación del sujeto con las tecnologías y la gubernamentalidad hegemónicas y, en consecuencia, 
a la relación ético-política a partir de la cual el sujeto se constituye como sujeto de una resistencia política.

\section{A modo de conclusión}

Siguiendo la iniciativa de Collier, al proponer una interpretación topológica del análisis de las relaciones de poder, propusimos realizar una interpretación topológica de las tres definiciones de política aquí analizadas, según la cual la tercera definición sobredetermina las dos anteriores, en cuanto permite pensar el modo en que tanto la relación bélica entre sujetos antagónicos cuanto la relación de resistencia ante una tecnología de gobierno, está siempre mediada históricamente por las relaciones estratégicas entre gubernamentalidades heterogéneas.

En consecuencia, desde esta perspectiva, la política no puede ser reducida a una relación antagónica entre adversarios, ni a una relación antagónica de resistencia- entre una forma de subjetivación y una gubernamentalidad, sino que más bien ambas definiciones están sobredeterminadas por la definición de política como relación estratégica entre gubernamentalidades heterogéneas. En efecto, por esto es que puede afirmarse que tanto la identidad que se constituye a partir de la identificación y el combate del enemigo, cuanto la que tiene lugar frente a una situación de dominación determinada, están mediadas por la conformación histórica de una gubernamentalidad hegemónica, contingente e inestable, sostenida en un juego de ajustes y combates específicos entre artes y tecnologías de gobierno, es decir, potencialmente confrontada a partir del recurso a la serie de saberes locales sometidos y descalificados, así como a las memorias de las luchas en las que se apoyarán los antagonismos políticos a los que el trabajo foucaultiano de crítica histórica erudita pretende aportarles un mapa posible del territorio histórico político en el que se despliegan.

No parece otra la tarea última perseguida por el diagnóstico del presente, definido como una ontología histórica de nosotros mismos que debiera ser entendida como la "crítica práctica en la forma del franqueamiento posible" (Foucault, 1999, p. 348), a partir de la determinación de los límites y los peligros específicos que forman parte de una situación hegemónica concreta y la abren a posibilidades de resistencia que adopten una "forma específica, en función del tipo y de la forma concreta que adopta en cada caso la dominación" (Foucault, 1996, p. 112). 


\section{Referencias}

Agamben, G. (2002). Homo Sacer I. El poder soberano y la nuda vida. (Trad. A. Gimeno Cuspinera). Madrid: Editora Nacional.

Agamben, G. (2005). Movement. Multitudes. Revue politique, artistique, philosphique. Revisado el 10 de marzo de 2017 en http://www.multitudes. net/movement/

Agamben, G. (2008). El Reino y la Gloria. (Trad. A. Gimeno Cuspinera). Buenos Aires: Pre-textos.

Agamben, G. (2006). El tiempo que resta. (Trad. A. Piñero). Madrid: Trotta.

Bidet, J. (2006). Foucault y el liberalismo: racionalidad, revolución, resistencia. Argumentos, 19(52), 1-27.

Blengino, L. (2017). Cuestiones de método: la grilla de inteligibilidad epocaltopológica y la lógica estratégica de las correlaciones y sistemas de dominantes en la historia política de la gubernamentalidad en los cursos de Michel Foucault. Ágora Filosófica, Revista de la Universidad Católica de Pernambuco, 17/1, jan/jun.

Brossat, A. (1994). La question de la plebe. A. Brossat (Ed.), Michel Foucault: les jeux de la vérité et du pouvoir (pp. 143-150). Nancy: Presses Universitaires de Nancy.

Castro-Gómez, S. (2012). Historia de la gubernamentalidad. Razón de Estado, liberalismo y neoliberalismo en Michel Foucault. Bogotá: Siglo del Hombre Editores.

Collier, S. (2009). Topologies of Power. Foucault's Analysis of Political Government beyond 'Governmentality'. Theory, Culture \& Society, 26(6), 78-108.

Ewald, F. (1999). Foucault and the contemporary scene. Philosophy \& Social Criticism, 25(3), 81-91.

Ewald, F. (2016). Foucault \& Neoliberalism. Revisado el 23 de agosto de 2016, en http://blogs.law.columbia.edu/foucault1313/2016/01/24/ewaldneoliberalism/

Foucault, M. (1992). Poderes y estrategias. Microfisica del poder (pp. 173-184). Madrid: Ediciones de La Piqueta.

Foucault, M. (1996). La ética del cuidado de uno mismo como práctica de la libertad. Hermenéutica del sujeto, 93-125. La Plata: Altamira.

Foucault, M. (1999). ¿Qué es la Ilustración? Estética, Ética y Hermenéutica. Obras esenciales, Vol. III, 335-352. Barcelona: Paidós.

Blengino, L. (2019). La política entre la guerra, la resistencia y la gubernamentalidad según Michel Foucault. Cuestiones de Filosofía, 4 (23), 47-74. 
Foucault, M. (2000a). Defender la sociedad. (Trad. H. Pons). Buenos Aires: Fondo de Cultura Económica.

Foucault, M. (2000b). Historia de la sexualidad I: La voluntad de saber. (Trad. U. Guiñazú). México: Siglo XXI.

Foucault, M. (2001a). El sujeto y el poder. Michel Foucault: más allá del estructuralismo y la hermenéutica, 241-259. Buenos Aires: Nueva Visión.

Foucault, M. (2001b). Dits et Écrits II. Paris: Gallimard.

Foucault, M. (2006). Seguridad, territorio, población. (Trad. H. Pons). Buenos Aires: Fondo de Cultura Económica.

Foucault, M. (2007). Nacimiento de la biopolítica. (Trad. H. Pons). Buenos Aires: Fondo de Cultura Económica.

Foucault, M. (2009). El gobierno de sí y de los otros. (Trad. H. Pons). Buenos Aires: Fondo de Cultura Económica.

Lemke, Th. (2002). Foucault, governmentality, and critique. Rethinking Marxism, 14(3), 49-74.

Locke, J. (2002). Segundo ensayo sobre el gobierno civil. (Trad. C. Piña). Buenos Aires: Losada.

Marchart, O. (2007). Post-Foundational Political Thought. Edinburgh: Edinburgh University Press.

Nosetto, L. (2014). Michel Foucault y la politica. San Martín: UNSAM Edita.

Raffin, M. (2018). La noción de política en la filosofía de Michel Foucault. Hermenéutica Intercultural. Revista de Filosofía, No 29, 29-59.

Rancière, J. (2000). Biopolitique ou politique. Multitudes. Revue politique, artistique, philosphique, $\mathrm{N}^{\circ} 1$, mars 2000. Revisado el 20 de marzo de 2017 en http://www.multitudes.net/Biopolitique-ou-politique/

Rancière, J. (2002). Peuple ou multitudes? Multitudes. Revue politique, artistique, philosphique, $\mathrm{N}^{\circ}$ 9, mai-juin 2009. Revisado el 20 de marzo de 2017 en http:// www.multitudes.net/Peuple-ou-multitudes/

Rancière, J. (2010). Momentos políticos. Buenos Aires: Capital intelectual.

Schmitt, Carl. (2004). El concepto de lo político. Carl Schmitt, teólogo de la política, 67-223. México: Fondo de Cultura Económica.

Senellart, M. (2006). Situación de los cursos. Seguridad, territorio, población, 417453. Buenos Aires: Fondo de Cultura Económica. 